University of Nebraska - Lincoln

DigitalCommons@University of Nebraska - Lincoln

Anthony F. Starace Publications

Research Papers in Physics and Astronomy

August 1976

\title{
The Quantum Defect Theory Approach
}

Anthony F. Starace

University of Nebraska-Lincoln, astarace1@unl.edu

Follow this and additional works at: https://digitalcommons.unl.edu/physicsstarace

Part of the Physics Commons

Starace, Anthony F., "The Quantum Defect Theory Approach" (1976). Anthony F. Starace Publications. 145. https://digitalcommons.unl.edu/physicsstarace/145

This Article is brought to you for free and open access by the Research Papers in Physics and Astronomy at DigitalCommons@University of Nebraska - Lincoln. It has been accepted for inclusion in Anthony F. Starace Publications by an authorized administrator of DigitalCommons@University of Nebraska - Lincoln. 
Published in PHOTOIONIZATION AND OTHER PROBES OF MANY-ELECTRON INTERACTIONS, ed. F. J. Wuilleumier (New York: Plenum, 1976).

THE QUANTUM DEFECT THEORY APPROACH

\author{
Anthony F. Starace* \\ Behlen Laboratory of Physics \\ The University of Nebraska, Lincoln, NE 68588, U.S.A.
}

The Quantum Uefect Theory (QUT) is a method of using the analytically known properties of excited electrons moving in a pure coulomb field to describe atomic photoabsorption and electron-ion scattering processes in terms of a few parameters. These parameters may be determined either from experimental data or from ab initio theoretical calculations. In addition, they are usually nearly independent of energy in the threshold energy region (i.e., within a few eV of the atomic ionization threshold). Thus the determination of these parameters at any single energy suffices to predict the variation with energy of numerous atomic properties in the threshold energy region such as total and partial photoionization or scattering cross sections, photoelectron asymmetry parameters, discrete line strengths, autoionization profiles, etc. These properties are often very strongly energy-dependent and difficult to measure or to calculate by other methods. Yet all these phenomena, according to the QUT, depend on only a few essential parameters which represent the proper interface between theory and experiment. The determination of these parameters should thus be the goal of both theory and experiment rather than the calculation or measurement of the various phenomena dependent on these parameters.

This article aims to describe the essence of the QDT for the non-specialist. More extensive surveys of the theory for the nonspecialist by Seaton ${ }^{1}$ and Fano $^{2}$ should be consulted for more complete references to the original literature. Here we shall first discuss the one channel theory since it embodies the main content of the general multichannel theory. Then we shall sketch the multichannel treatment of Lu and Fano and its applications.

*Alfred P. Sloan Foundation Fellow 
In common with the closely related R-matrix theory, the QDT assumes that the configuration space for an excited atomic electron can be divided into two regions: an inner region, $0 \leq r \leq r$, where electron correlations are strong and difficult to treat? and an outer region, $r \leq r \leq \infty$, where the electron-ion interaction potential is assumed to be purely coulombic and where the form of the electron wavefunction is known analytically. The boundary radius $r$ between the two regions is typically of the order of the atomic radius: one wants $r$ as small as possible so that the electron wavefunction can be knowh exactly over as great a range of $r \geq r$ as possible and yet one wants $r$ large enough so that the approximation of a pure coulomb field f8r $r \geq r$ makes sense. (The inclusion of $r^{-2}$ long range potentials for $P_{2 r_{0}}$ has been treated by Bely. ${ }^{3}$ )

Consider now the one-channel problem of an excited electron in an alkali atom: the electron sees a Coulomb field for $r \geq r$, where $r$ is roughly the ionic radius. We measure the energy $\varepsilon$ of the excited electron relative to the ionization threshold and make the change of variables $\varepsilon=-0.5 \nu^{-2}$. The parameter $v$ will thus be our measure of energy. The Schrddinger equation for $r \geq r$ has two solutions, one regular and one irregular for smalt values of $r$ :

$$
\begin{array}{ll}
f(\nu, r) \sim r^{\ell+1} & \text { as } r \rightarrow 0 \\
g(\nu, r) \sim r^{-\ell} & \text { as } r \rightarrow 0
\end{array}
$$

A general solution of the Schrodinger equation for $r \geq r$ is a linear combination of $f(\nu, r)$ and $g(\nu, r)$ with coefficients to Be determined by application of boundary conditions at infinity and at $r_{0}$. The form of this general solution turns out to be

$$
\psi(\nu, r)=N_{\nu}\{f(\nu, r) \cos \pi \mu-g(\nu, r) \sin \pi \mu\} \text { for } r \geq r_{0}
$$

where $N_{\text {is }}$ is normalization factor which is determined by the behavior of $\psi(\nu, r)$ at large $r$. $\mu$, on the other hand, is the relative phase with which the regular and irregular solutions are superposed. Its value is determined by the behavior of $\psi(\nu, r)$ in the core region, $0 \leq r \leq r$, where the effective potential is non-Coulombic: j.e., $\mu$ has that value which allows the analytically determined $\psi(\nu, r)$ given by Eq. (2) for $r \geq r_{0}$ to be joined smoothly at $r=r_{0}$ onto the numerically determined portion of $\psi(\nu, r)$ that obtains in the inner core region, $0 \leq r \leq r$, which we shall call $\psi_{g}(\nu, r)$. (Thus $\psi(\nu, r)$ without other specification is assumed to be the electron wavefunction over all space; $\psi(\nu, r)$ is given by Eq. (2) for $r \geq r_{0}$ and by $\psi_{c}(\nu, r)$ for $\left.0 \leq r \leq r_{0} \cdot\right)$

$A b$ initio determination of the parameter $\mu$ at any energy requires numerical calculation of the inner core wavefunction $\psi_{\mathrm{f}}(\nu, r)$. The R-matrix theory ${ }^{4,5}$ is designed for this. The procedure is to 
compute the complete set of discrete eigenfunctions $u_{\lambda}(r)$ for the spherical "box" $0 \leq r \leq r$. The inner core wavefunction for energy $v$ is then represented as a linear combination of this complete set of eigenfunctions:

$$
\psi_{c}(\nu, r)=\sum_{\lambda} c_{\lambda}(\nu) u_{\lambda}(r)
$$

The coefficients $c_{\lambda}(\nu)$ are determined by the R-matrix theory to depend on energy $\nu$ and on values of $\psi_{c}(\nu, r)$ and $u_{\lambda}(r)$ and their derivatives at $r=r$. Requiring the logarithmic derivatives of Eqs. (2) and (3) t8 be equal at $r=r$ permits the determination of $\mu .{ }_{4}-{ }_{6}$ Other more approximate methods may also be used to calculate $\mu$ : e.g., if a model potential $V(r)$ is used to describe the electron's motion in the region $0 \leq r \leq r_{0}$, then $\mu$ may be calculated by the Phase-Ámplitude Method.

Alternatively, $\mu$ may be determined semi-empirically and, provided sufficient empirical data are available, there may be no need to know the inner core wavefunction in order to predict the variation with energy of various atomic properties in the threshold energy region. Specifically, consider the asymptotic behavior of $\psi(\nu, r)$ in the case of excited electron energies below threshold, i.e., $\psi(\nu, r)$ must tend toward zero. The asymptotic forms of the regular and irregular Coulomb functions are: ${ }^{8}$

$$
\begin{array}{ll}
f(\nu, r) \rightarrow u(\nu, r) \sin \pi \nu-v(\nu, r) \operatorname{expi\pi } v & \text { as } r \rightarrow \infty \\
g(\nu, r) \rightarrow-u(\nu, r) \cos \pi \nu+v(\nu, r) \operatorname{expi} \pi\left(\nu+\frac{1}{2}\right) & \text { as } r \rightarrow \infty
\end{array}
$$

where $u(v, r)$ is an exponentially increasing function of $r$ and $v(\nu, r)$ is an exponentially decreasing function of $r$. Substituting Eq. (4) in Eq. (2) gives:

$$
\psi(\nu, r) \rightarrow N_{\nu}\{u(\nu, r) \sin \pi(\nu+\mu)-v(\nu, r) \operatorname{expi\pi }(\nu+\mu)\} \text { as } r \rightarrow \infty
$$

In order that $\psi(v, r)$ tend toward zero the coefficient of $u(v, r)$ must be zero; i.e. $\sin \pi(\nu+\mu)=0$ or $\nu+\mu=n$, where $n$ is an integer. Substituting $\nu=n-\mu$ in the expression for the electron's energy gives

$$
\varepsilon=-\frac{1}{2 v^{2}}=-\frac{1}{2(n-\mu)^{2}}
$$

$\mu$ is thus the quantum defect of spectroscopy and may be determined directly from Rydberg energy level data for the alkalis.

For positive excited electron energies, on the other hand, $v=i / k$, where $k$ is the electron momentum. The asymptotic forms of the regular and irregular coulomb functions are: ${ }^{8}$ 


$$
\begin{aligned}
& f(\nu, r) \rightarrow\left(\frac{2}{\pi k}\right)^{\frac{1}{2}} \sin (k r+\theta) \text { as } r \rightarrow \infty \\
& g(\nu, r) \rightarrow-\left(\frac{2}{\pi k}\right)^{\frac{1}{2}} \cos (k r+\theta) \text { as } r \rightarrow \infty
\end{aligned}
$$

where

$$
\theta=-\frac{1}{2} \ell \pi+\frac{1}{k} \ln (2 k r)+\arg \Gamma(\ell+1-i / k) .
$$

Substituting Eq. (7) in Eq. (2) gives

$$
\psi(k, r) \rightarrow N_{k}\left(\frac{2}{\pi k}\right)^{\frac{3}{2}} \sin (k r+\theta+\pi \mu) \text { as } r \rightarrow \infty
$$

Eq. (8) implies that $\pi \mu$ is the scattering phase shift for the continuum electron at energies near threshold.

Seaton 9,10 first showed this connection, via $\mu$, between discrete energy level data and scattering phase shift data. Subsequent work by Seaton and collaborators ${ }^{1}$ used empirical energy level data to obtain electron-ion scattering phase shifts, and, conversely, ab initio calculated phase shifts to obtain the quantum defects. Lu, Fano, and collaborators ${ }^{2}$ have developed alternative methods, described below, for obtaining QDT parameters such as $\mu$ from empirical energy level data, and have begun the ab initio calculation of these parameters by the R-matrix theory. ${ }^{6}$

The theory described above would be in vain if $\mu$ were a rapidly varying function of energy since then separate calculations would be needed at each energy. Fortunately $\mu$ is a slowly varying function of energy since it is determined from the inner core wavefunction $\psi_{(}(\nu, r)$, which except for a normalization factor is insensitive to smail changes in the electron's energy. This insensitivity is due to the electron's large kinetic energy in the inner core region. From Eq. (2) we can see directly that for small $r \geqslant r$, $\psi(v, r)$ depends on energy mainly through the normalization factor $N$ since $\mu$ is weakly energy-dependent and so are $f(\nu, r)$ and $g(v, r)$ at small $r$ (cf. Eq. (1)). The normalization factor $W_{v}$ is determined by the asymptotic behavior of $\psi(\nu, r)$ and may be very energy-dependent. The point of this discussion thus is that at small radii $\psi(\nu, r) / N_{v}$ is likely to be quite insensitive to energy in the threshold energy region and this is one reason why the predictions of the QDT are often so uncannily good.

To show how energy-independent is the form of $\psi(\nu, r)$ for sma 11 $r$, consider Table I, which presents data for the $n s(1 \leq n \leq 7)$ bound wavefunctions of atomic uranium. ${ }^{1}$ Notice that as the orbital energies increase there is a remarkable convergence of the positions of the first maxima, first minima, and the first two nodes of the 
radial wavefunctions to energy-independent values. We have also arbitrarily re-normalized each one of the seven radial wavefunctions to unity at the position of its first maximum by dividing $P_{n s}(r)$ by $P_{n s}\left(r_{M A X}\right)$, where PMAXI is the position of the first maximum. This re-normalization should eliminate any energy dependence of the wavefunction amplitude arising from the normalization factor. The last column of Table I shows the re-normalized amplitudes at the positions of the first minima of the wavefunctions. Once again, as the orbital energies increase there is a convergence of the re-normalized amplitudes to an energy-independent value. Note that Table I includes only unexcited electron orbitals since these are tabulated in readily available references. 11 QDT, however, is concerned with excited electron orbitals having energies within a few eV of threshold. Table I shows clearly (cf. the 6s and 7s orbitals) that over a range of a few eV the form of $\psi(\nu, r)$ for small $r$ is to an excellent approximation energy-independent or at most very weakly dependent on energy.

Direct applications of the insensitivity of $\psi(\nu, r) / N_{\psi}$ upon energy are readily made and serve to indicate the power of the QDT. Consider the calculation of discrete oscillator strengths for transitions to members of a one-channel series of Rydberg levels. The oscillator strength $f_{n}$ is proportional to the square of the electric dipole matrix element, whose radial part is:

$$
R_{v}=\int_{0}^{\infty} r^{2} d r \psi_{0}(r) r \psi(\nu, r)
$$

where $\nu=(n-\mu)$ and $\psi_{0}(r)$ is the electron's initial state wavefunction, whose range is comparable to $r_{0}$. Over the effective range of inte-

TABLE I: Behavior of Uranium ns Radial Wavefunctions

$$
P_{n s}(r) / r \text { for Smal1 Radial Distances } r \text {. }
$$

Positions (in Bohr) of the first maximum $\left(r_{\text {MAXI }}\right)$, first minimum $\left(r_{\text {MINI }}\right)$, and first two nodes $\left(r_{\text {NODE } 1,2}\right)$ of
$P_{n s}(r)$ :

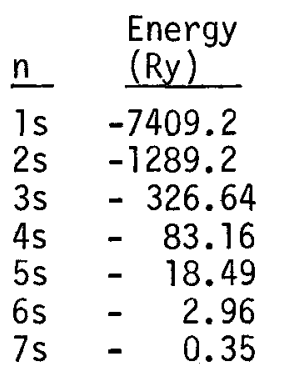

\begin{tabular}{ll}
$r_{\text {MAX1 }}$ & $r_{\text {NODE1 }}$ \\
\hline 0.01097 & $-\cdots-\overline{0}$ \\
0.00844 & 0.02199 \\
0.00823 & 0.02110 \\
0.00817 & 0.02089 \\
0.00816 & 0.02083 \\
0.00815 & 0.02082 \\
0.00815 & 0.02082
\end{tabular}

\begin{tabular}{l}
$r_{M I N 1}$ \\
\hline 0.05922 \\
0.04771 \\
0.04620 \\
0.04583 \\
0.04572 \\
0.04581
\end{tabular}

\begin{tabular}{l}
$r_{\text {NODE2 }}$ \\
\hline-0.08230 \\
0.07812 \\
0.07714 \\
0.07691 \\
0.07686
\end{tabular}

$P\left(r_{\text {MIN1 }}\right)$

$\underline{P\left(r_{\text {MAXI }}\right)}$

$-1.9589$

$-1.6643$

$-1.6223$

$-1.6128$

$-1.6095$

$-1.6093$ 
gration, however, $\psi(\nu, r)$ depends on energy mainly through $N_{\nu}$. Hence $R_{\nu} / N_{\nu}$ is only weakly energy-dependent, or, equivalently, $\mathrm{f}_{n} / \mathrm{N}_{v}{ }^{2}$ is only weakly energy-dependent.

It can be shown ${ }^{7}$ that $N_{\nu}$ is proportional to $v^{-3 / 2}=(n-\mu)^{-3 / 2}$ for discrete (i.e., negative) electron energies and that $N_{\nu}$ is independent of energy for positive electron energies (assuming normalization per unit energy). This implies that multiplication of the discrete oscillator strength $f$ by $(n-\mu)^{3}$ will produce a spectrum of oscillator strengths that (1) varies slowly from one discrete level to another and (2) joins smoothly onto the continuous spectrum of oscillator strength. (This "renormalization" serves to give the discrete final state wavefunctions continuumtype normalizations so that the oscillator strength is then continuous across the ionization threshold. ${ }^{12}$ )

Fig. 1 illustrates this renormalization procedure for $\mathrm{H}$ and Li discrete and continuous oscillator strengths. The area of each rectangle corresponds to the value of the discrete oscillator strength $f_{n}$ ( $n$ is labeled $s$ in the figure). The height of each rectangle equals $(n-\mu)^{3} f_{n}$. It is seen that when plotted in this way the discrete oscillator strength joins smoothly onto the continuous oscillator strength at threshold. The deviation from constancy near threshold is due to the residual, weak energy dependence

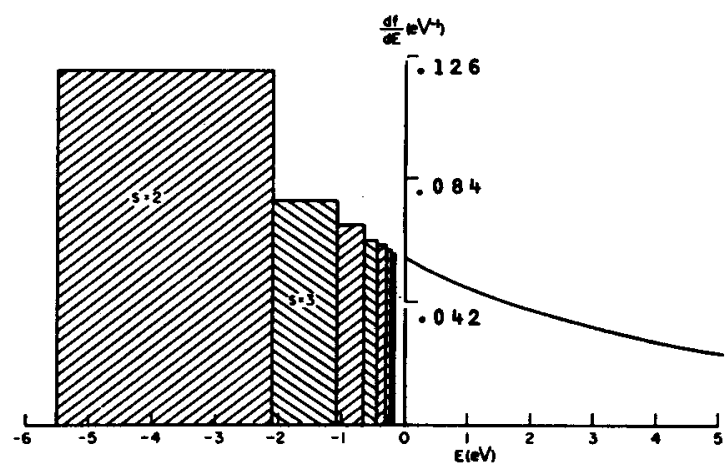

(a)

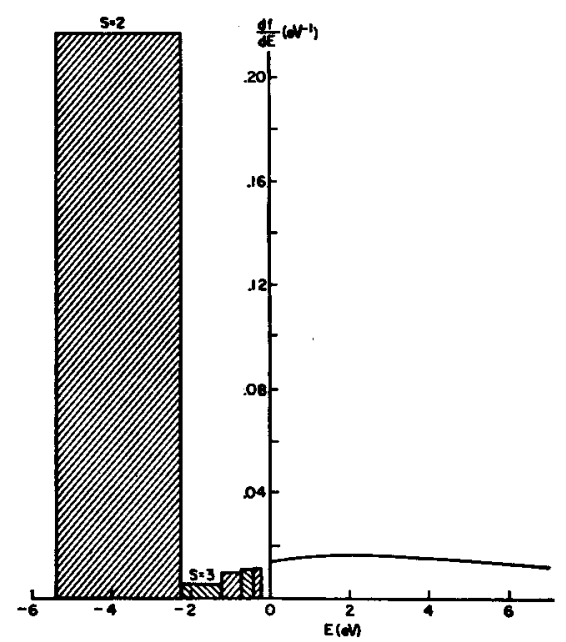

(b)

Fig. 1. Oscillator strength distribution in the discrete and part of the continuous spectra of (a) H (theory) (b) Li (experiment). (From Ref. 12) 
of the radial dipole matrix elements and possibly, in the case of $L i$, of the quantum defect $\mu$. The QDT often takes account of the weak energy dependence of these parameters by expanding them in a power series in energy about their value at threshold and keeping only the first two or three terms. 13,14

The QDT for the one-channel (alkali-like) spectra thus describes the discrete energy spectrum by the quantum defect $\mu$, which is determined by electron-ion interactions in the inner core region, and by the position of the ionization threshold. The electron-ion scattering phase shift near threshold is equal to $\pi \mu$. In addition, knowledge of the oscillator strength at a single energy near threshold permits the prediction of all discrete and continuous oscillator strengths in the threshold energy region.

Consider now the multi-channel case of rare gas photoabsorption spectra. There are five channels, which in $j j$-coupling are specified as follows:

$$
\begin{aligned}
p^{6}\left({ }^{1} S_{0}\right)+h \nu & \rightarrow p^{5}\left({ }^{2} p_{3 / 2}\right) \varepsilon d_{5 / 2} J=1 \\
& \rightarrow p^{5}\left({ }^{2} P_{3 / 2}\right) \varepsilon d_{3 / 2} J=1 \\
& \rightarrow p^{5}\left({ }^{2} P_{3 / 2}\right) \varepsilon s_{1 / 2} J=1 \\
& \rightarrow p^{5}\left({ }^{2} P_{1 / 2}\right) \varepsilon d_{3 / 2} \mathrm{~J}=1 \\
& \rightarrow p^{5}\left({ }^{2} P_{1 / 2}\right) \varepsilon s_{1 / 2} \mathrm{~J}=1
\end{aligned}
$$

In Eq. (10) $\varepsilon$ indicates the excited electron's energy, which may be positive or negative (i.e., discrete). jj-coupling is appropriate at large $r$, when the ion core and the excited electron are far apart. At such large distances the difference between the two ionization thresholds $\mathrm{I}_{3 / 2}$ and $\mathrm{I}_{1 / 2}$ corresponding to the two levels of the ion dominates the electron-ion core interaction. If these five channels were not interacting one would expect each one to have a characteristic energy-independent quantum defect, just as though each channel could be considered a one-channel case. In fact, at short range the electrostatic interaction between the excited electron and the ion core is dominant and the difference in ionic thresholds pales in significance compared to the excited electron's large kinetic energy. This short range interaction results in what spectroscopists call series perturbations, i.e., quantum defects that vary dramatically along a Rydberg series due to the interaction between nearby levels of different Rydberg series.

The multi-channel QDT treats the electron-ion interaction in the rare gases thusly: ${ }^{14,} 15$ at large distances $r$ the five excited electron channels are described by the asymptotic coupling appro- 
priate to the interactions remaining at large distances, i.e., jj-coupling as in Eq. (10). These asymptotic channel states are not eigenstates of the electron-ion interaction at small distances, but instead interact. To take a scattering theory point of view, one says that an asymptotic channel state is scattered, by interactions predominant in the inner core region $0 \leq r \leq r$, into other asymptotic channel states. If we label two such asymptotic states by $i$ and $j$, then $S_{i j}$ indicates the appropriate scattering matrix element arising from short range interactions. This matrix element may be cast in terms of the diagonal representation of $S$ as follows:

$$
S_{i j}(J=1)=\sum_{\alpha=1}^{5} U_{i \alpha} e^{2 i \pi \mu} \alpha U_{\alpha j}^{\dagger} \quad(i, j=1,5)
$$

Here $\alpha$ labels the five scattering eigenstates of the short range electron-ion interaction. In the rare gases these eigenstates have been found to be fairly close to the LS-coupled states of the ion and the excited electron due to the dominance of electrostatic interactions at short range. The unitary matrix $U_{j \alpha}$ is the matrix that diagonalizes $S_{j j}(J=1)$. It represents the transformation from the scattering eigenstates $\alpha$ to the asymptotic, experimentally observed states $i . U_{j \alpha}$ is thus called the "frame transformation matrix," because it transforms from the strong interaction frame appropriate to the inner core region to the weak interaction frame appropriate to the asymptotic region. The parameters $\mu_{\alpha}$ are the eigenphase shifts of the scattering eigenstates $\alpha$ : i.e., $\mu_{\alpha}$ is the phase by which the eigenstate $\alpha$ is shifted in traversing the inner core region.

In the multi-channel QDT for the rare gases the final state of the excited electron-ion core system is characterized by the eigenphase shifts $\mu_{\alpha}$, the ionization thresholds $I_{3} /{ }^{2}$ and $I_{1} /{ }^{2}$, and the frame transformation matrix $U_{i \alpha}$. It is possible, as ih the onechannel case, to obtain these parameters by ab initio theoretical calculations, as for example by use of the multi-channel R-matrix theory. ${ }^{6}$ It remains for us in this article, however, to show how these parameters are related to experimental energy level data and thus how these parameters may be determined semi-empirically.

As in the one-channel case, the final state wavefunction for the excited rare gas system may be written analytically in the region $r>r$. Since there are two ionic energy levels (i.e., ${ }^{2} \mathrm{P}$ and $\left.{ }^{2} \mathrm{P}_{1(2}\right)^{\circ}$ we must include the ionic wavefunction explicitiy bétause for a given excitation energy $E$ there correspond two excited electron energies $\varepsilon_{i}$ :

$$
E=I_{i}+\varepsilon_{i}=I_{i}-\left(2 v_{i}^{2}\right)^{-1}(j=3 / 2,1 / 2)
$$

Superposing the regular and irregular Coulomb functions in each 
channel and summing over the allowed channels gives for the final state wavefunction:

$$
\begin{aligned}
\psi & =\sum_{\alpha} \psi_{\alpha} A_{\alpha}= \\
& =\sum_{i} \Phi_{i}\left\{f\left(\nu_{i}, \ell_{i} ; r\right) \sum_{\alpha} U_{i \alpha} \cos \pi \mu_{\alpha} A_{\alpha}-g\left(\nu_{i}, \ell_{i} ; r\right) \sum_{\alpha} U_{i \alpha} \sin \pi \mu_{\alpha} A_{\alpha}\right\}
\end{aligned}
$$

In Eq. (13) $\ell_{j}$ denotes the electron's orbital momentum in channel $i, \Phi_{j}$ represents the ionic wavefunction as well as the angular and spin variables of the excited electron, and the coefficients $A_{\alpha}$ represent the weights with which the scattering eigenstates $\psi_{\alpha}$ are superposed. The coefficients $A_{\alpha}$ are determined by application of one of the three types of boundary condition at infinity appropriate for $\psi$, corresponding to each of the following three energy regions: EsI $I_{3 / 2}$ (the discrete region), $I_{3}{ }_{2} \leq E \leq I_{3} / 2$ (the autoionizing region), ${ }^{3} 1_{1 / 2}^{2} \leq E$ (the photoionization ${ }^{3}$ region)! For example, in the discrete energy region $\psi$ must tend toward zero at large $r$. Substituting the asymptotic forms for $f$ and $g$ given by Eq. (4) into Eq. (13) and requiring the coefficients of the exponentially increasing functions $u\left(v_{j}, r\right)$ to be zero gives

$$
\sum_{\alpha} U_{i \alpha} \sin \pi\left(v_{i}+\mu_{\alpha}\right) A_{\alpha}=0
$$

which in turn implies that non-trivial solutions for $A_{\alpha}$ exist only if

$$
F\left(v_{3 / 2}, v_{1 / 2}\right) \equiv \operatorname{det}\left|u_{i \alpha} \sin \pi\left(v_{i}+\mu_{\alpha}\right)\right|=0 \text {. }
$$

Lu and Fano $8,15,16$ have developed the requisite procedures for determining the eigenphases $\mu_{\alpha}$ and portions of the frame transformation matrix $U_{\text {ja }}$ by fitting $E q$. (15) to experimental energy level data. In the present case of two ionic thresholds, this fitting procedure is best considered with reference to the two-dimensional Lu-Fano plot, ${ }^{16}$ although the procedure is no means limited to two threshold problems. A typical Lu-Fano plot is given in Fig. 2 for Ar Rydberg energy levels belonging to the five series of Eq. (10). For each experimental term level E, regardless of the spectroscopic classification of the level, the effective quantum numbers $\nu_{3} / 2$ and $\nu_{1 / 2}$ are calculated from $\mathrm{Eq}$. (12). Since the function in $\mathrm{Eq}^{3}$ ( $/ 2 \mathrm{~T}$ ) is invariant to integer changes in the values of $v_{i}(i=3 / 2,1 / 2)$, these are only calculated modulo 1. (In treating the energy-dependence of the parameters $\mu_{\alpha}$ and $U_{j \alpha}$, however, the absolute values of $\nu_{j}$ must be used ${ }^{13,14} \ldots$ we ignore this complication here and consider only levels close to threshold, where the parameters are energyindependent.) The Lu-Fano plot is constructed by plotting $-\mathrm{V}_{3} / 2$ $(\bmod .1)$ vs. $v_{1 / 2}(\bmod .1)$ as shown in Fig. 2 .

Except for the lowest levels with $n \leqslant 6$, the resulting plot of 
levels falls on the solid curves, which are given by the function in Eq. (15) with appropriately fitted parameters $\mu_{\alpha}$ and $U_{i \alpha^{*}}$ This function is multivalued, having three horizontal branches, corresponding to the three series in Eq. (10) belonging to the ionic threshold, and two vertical portions corresponding to ${ }^{3}$ the two series in Eq. (10) belonging to the ${ }^{2} \mathrm{P}$ ionic threshold. Each branch of this function is seen to be monotonically increasing and, in fact, the entire function is continuous in the sense that at any edge of the unit square it is reflected to the opposite edge with the same value. It can also be shown that: ${ }^{15,16}(1)$ The five intersections of the solid line with the diagonal line partially drawn in Fig. 2 occur at the five values of the scattering eigenphases $\mu_{\alpha} ;(2)$ At these same five intersections, the slopes of the solid curve give information on the frame transformation matrix $U_{j \alpha}$. Fig. 2 demonstrates quite well the theoretical correlation of very many experimental energy level data by means of a very few parameters $\left(\mu_{\alpha}\right.$ and $\left.U_{i \alpha}\right)$.

In a similar manner, the intensities of the discrete absorption lines, the autoionizing level profiles, and the photoionization cross section at threshold may be determined in the multi-channel QDT by means of the $\mu_{\alpha}$, the $U_{j \alpha}$, and five parameters $D_{\alpha}, 1 \leq \alpha \leq 5$, corresponding to the radial electric dipole matrix elements for transitions from the ground state to the five eigenstates $\psi_{\alpha}$. The parameters $D_{\alpha}$ and portions of the $U_{j \alpha}$ matrix are determined by fitting analytic equations for intensities to available experimental data. In the paper by Lee and Lu, ${ }^{14}$ for example, these parameters were determined by fitting an experimental autoionization line profile. The parameters thus determined were then used to predict discrete oscillator strengths.

It is in this way that the dependence of disparate experimental data, occuring in different energy regions, on a few common, nearly energy-independent parameters is exploited by the QDT. An essential conceptual aspect of the multichannel theory is the connection of the asymptotic (observable) states to the strong-interaction scattering eigenstates by means of a frame transformation matrix $U_{j \alpha}$. In the case of molecules, ${ }^{8}$ this transformation is from the body frame of the molecule, appropriate at short range (where the excited electron closely follows the motion of the molecule), to the laboratory frame, appropriate asymptotically (where the rotational motion of the molecular ion only effects the excited electron's motion through their mutual energy sharing, which depends on the differing rotational energy levels of the molecular ion). In a similar way, negative ion photodetachment has been treated by multichannel QDT. ${ }^{17}$

So far all but one ${ }^{6}$ of the applications of the QDT by Lu, Fano, and collaborators ${ }^{2}$ and a11 applications for complex systems 


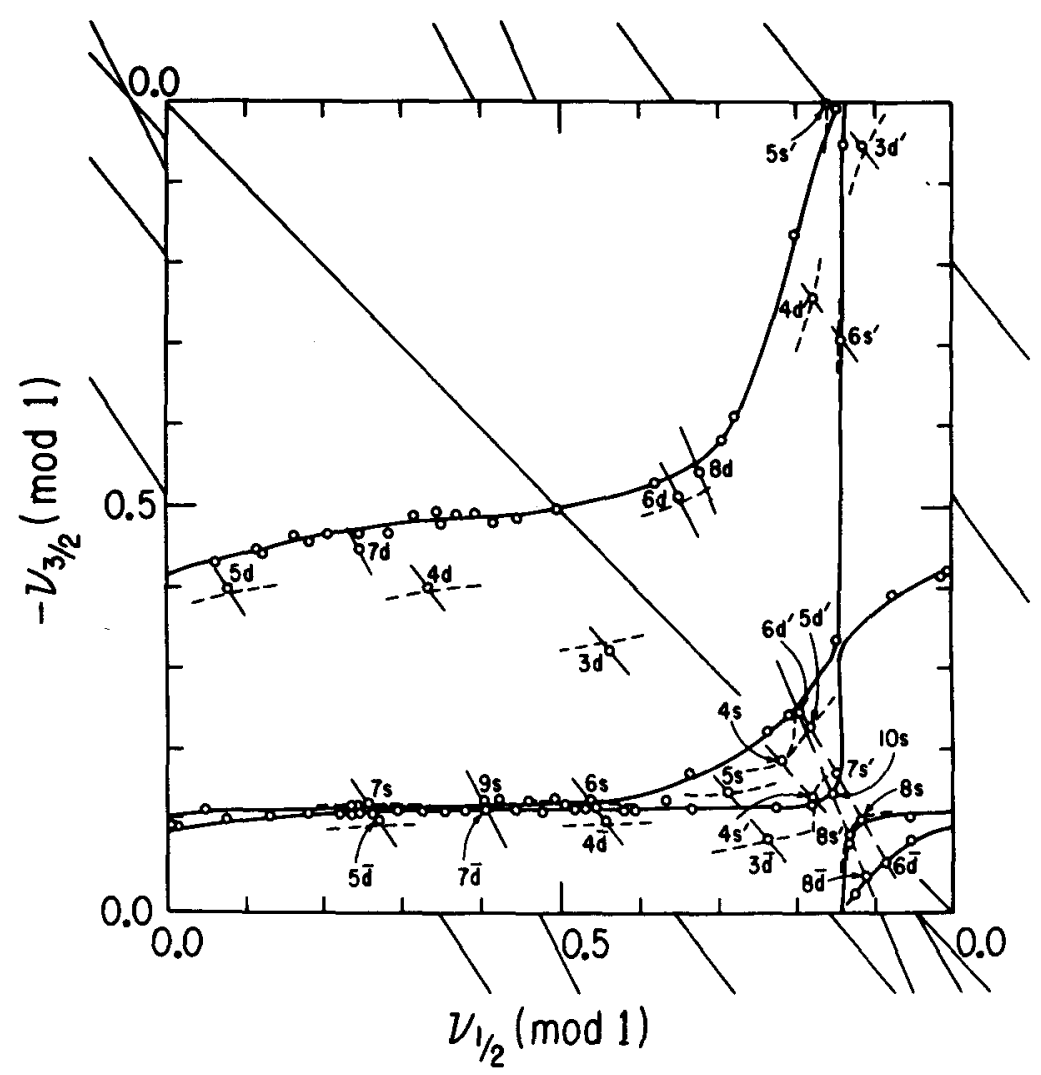

Fig. 2. $-v_{3} / 2(\bmod .1)$ vs. $v_{1 / 2}(\bmod , 1)$ : open circles represent experimenta ${ }^{3} \mathrm{Ar}^{2}$ energy levels; ${ }^{1}{ }^{2}$ lid curves represent $\mathrm{Eq}$. (15) with parameters $\mu_{\alpha}$ and $U_{i \alpha}$ determined by fitting energy level data near the $\mathrm{I}_{3 / 2}$ threshold. '(From Ref. 14)

by Seaton and collaborators ${ }^{1}$ have depended on the availability of reliable experimental data in order to obtain the necessary parameters in the theory. Seaton and collaborators have also determined the parameters by ab initio calculations, using closecoupling methods, for light atomic systems. Only recently, however, with the development of the R-matrix theory, is it practical to obtain the necessary parameters for heavy atoms by ab initio calculations. ${ }^{6}$ Much development of the theory still needs doing. It is worth doing because the focusing of theorists and experi- 
mentalists on obtaining the few scattering parameters needed for any atomic or molecular system can only lead eventually to greater unity and coherence in low-energy atomic physics and hopefully permit the treatment of presently intractable problems.

\section{References}

1. M. J. Seaton, Comments on Atomic and Molecular Physics II, 37 (1970).

2. U. Fano, J. Opt. Soc. Am 65, 979 (1975).

3. 0. Bely, Proc. Phys. Soc. (London) 88, 833 (1966).

4. G. Breit, Handbuch der Physik XLI/1, 1 (1959).

5. P. G. Burke and W. D. Robb, Adv. Atomic Mol. Phys. (to be published).

6. C. M. Lee, Phys. Rev. A 10, 584 (1974).

7. J. L. Dehmer and U. Fano, Phys. Rev A 2, 304 (1970).

8. U. Fano, Phys. Rev. A 2 , 353 (1970).

9. M. J. Seaton, Compt. Rend. 240, 1317 (1955).

10. M. J. Seaton, Mon. Not. Roy. Astron. Soc. 118, 504 (1958).

11. The data presented in Table I were obtained by interpolating the values tabulated by F. Herman and S. Skillman, Atomic Structure Calculations (Prentice-Hall, Englewood Cliffs, New Jersey, 1963).

12. U. Fano and J. W. Cooper, Rev. Mod. Phys. $\underline{40}, 441$ (1968), §2.4.

13. A. F. Starace, J. Phys. B 6, 76 (1973).

14. C. M. Lee and K. T. Lu, Phys. Rev. A $\underline{8}, 1241$ (1973).

15. K. T. Lu, Phys. Rev. A $\underline{4}, 579$ (1971).

16. K. T. Lu and U. Fano, Phys. Rev. A 2, 81 (1970).

17. A. R. P. Rau and U. Fano, Phys. Rev. A $\underline{4}, 1751$ (1971). 